\title{
Comparison of the Fundus and the Renal Findings in Spontaneously Hypertensive Rats
}

\author{
Shigeki TaKahashi
}

Fundus findings of 31 spontaneously hypertensive rats, consisting of 4 rats of $F_{14,15}$ rats of $F_{15}, 10$ rats of $F_{16}, 3$ rats of $F_{17}, 1$ rat of $F_{18}$, and 8 rats of $F_{19}$ were compared with histopathologic findings of the kidney.

The following results were obtained.

1. Retinal opacity or papilledema were observed in 21 rats out of 31 .

2. In the kidney, nephrosclerosis were observed in 4 rats out of 21 .

3. Such nephrosclerotic changes were more remarkable in cases of earlier onset of hypertension. 